\title{
UN AUDITORIO AL AIRE LIBRE EN LA ANTIGUA FERIA DEL CAMPO EN MADRID, ESPAÑA
}

\author{
(AN OPEN AUDITORIUM IN OLD «FERIA DEL CAMPO»IN MADRID/SPAIN)
}

Salvador Pérez Arroyo, Arquitecto

$146-98$

\section{RESUMEN}

Se presenta en este articulo el escenario y servicios complementarios construidos para acondicionar la Pista de Exposiciones de la Antigua Feria del Campo de Madrid, como recinto para conciertos Pop y otros espectáculos multitudinarios.

Su estudio despierta interés, no sólo por lo reciente de su realización, sino también por la tecnologia que para la misma se empleó, tanto por los plazos de ejecución, como por sus singulares y extremas condiciones de uso previstas: soporte de amplias y aparatosas tecnologias contemporáneas de la comunicación.

Entre las peculiaridades que caracterizan el proyecto destacan:

- La solución de acceso y evacuación del público; asi como de carga y descarga de mercancias, lo cual exige acondicionamiento del amplio entorno de influencia del recinto: modificación y construcción de edificaciones y pabellones de servicio adyacentes, junto con la jardineria.

- Las dimensiones de los elementos constructivos empleados, que han exigido el continuo estudio y modificación del proyecto a lo largo de su planteamiento en busca de los consabidos óptimos: función - economia - sencillez de planteamiento: $75 \mathrm{~m}$ de vano en una viga-puente.

- El proceso de montaje, que exigió una modulación de piezas operativa, exigió a la vez una independencia en la ejecución de los elementos constructivos.

\section{SUMMARY}

This work describes the stage and the complementary services carried out for the conditionning of the courtyard for expositions inside the "Feria del Campo" in Madrid, as a ground for Pop concerts and other multitudinous entertainments.

Its study awakes interest, not only by the newness of its construction but also by its technology, the lasting of execution as well as the peculiarity of its extreme conditions: a pillar of the huge and sophisticated contemporary tecnologies of communication.

It stands out among the peculiarities that characterize it:

- The solution for the entrance and exit of the public so as for the loading and unloading of the goods that makes cecassary the acommodation of the surroundings: modification and construction of pavillions and adjoined service buildings and gardens.

- The size of the constructive elements, which have been the object of a careful study, and the modification of the project along the search of the wellknown optimums: functionalism, economy, laying out simpleness: a clear span beam of $75 \mathrm{~m}$.

- The setting up process, that needed an operative modulation of the parts, needed at the same time independent constructive elements.

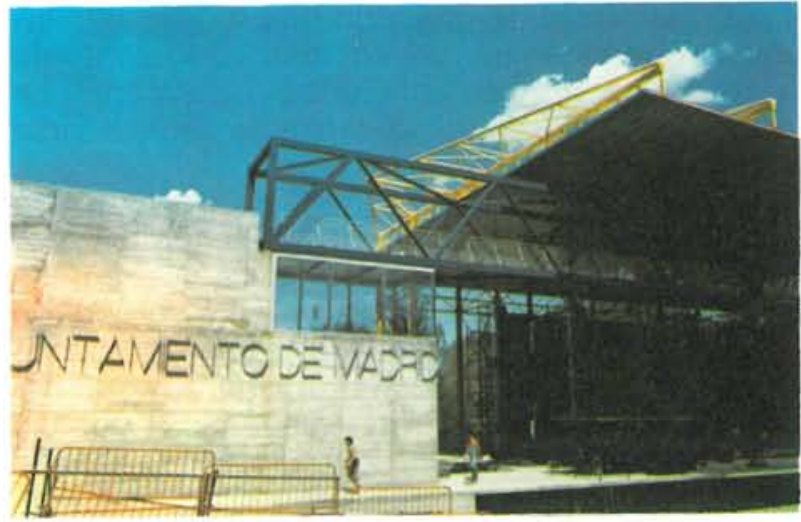

Aspecto del estribo izquierdo con la cabina de control bajo la pasarela.
En el Auditorio iniciado en la antigua Feria del Campo de Madrid, dentro de la Casa de Campo, se ha utilizado como base las gradas de un anfiteatro preexistente junto al espléndido Palacio de Cristal del arquitecto F. Cabrero.

El encargo inicial pretendia la construcción de un escenario fijo que sustituyese al provisional instalado todos los años en el madrileño parque del Oeste. El programa en un principio simple se reveló inmediatamente complejo: la asistencia masiva de hasta cien mil personas a este tipo de actuaciones, asi como las muy diversas exigencias que podian plantear los distintos espectáculos, nos condujeron a una solución que cerrando el amplio fondo del auditorio, permitiera una gran versatilidad. 


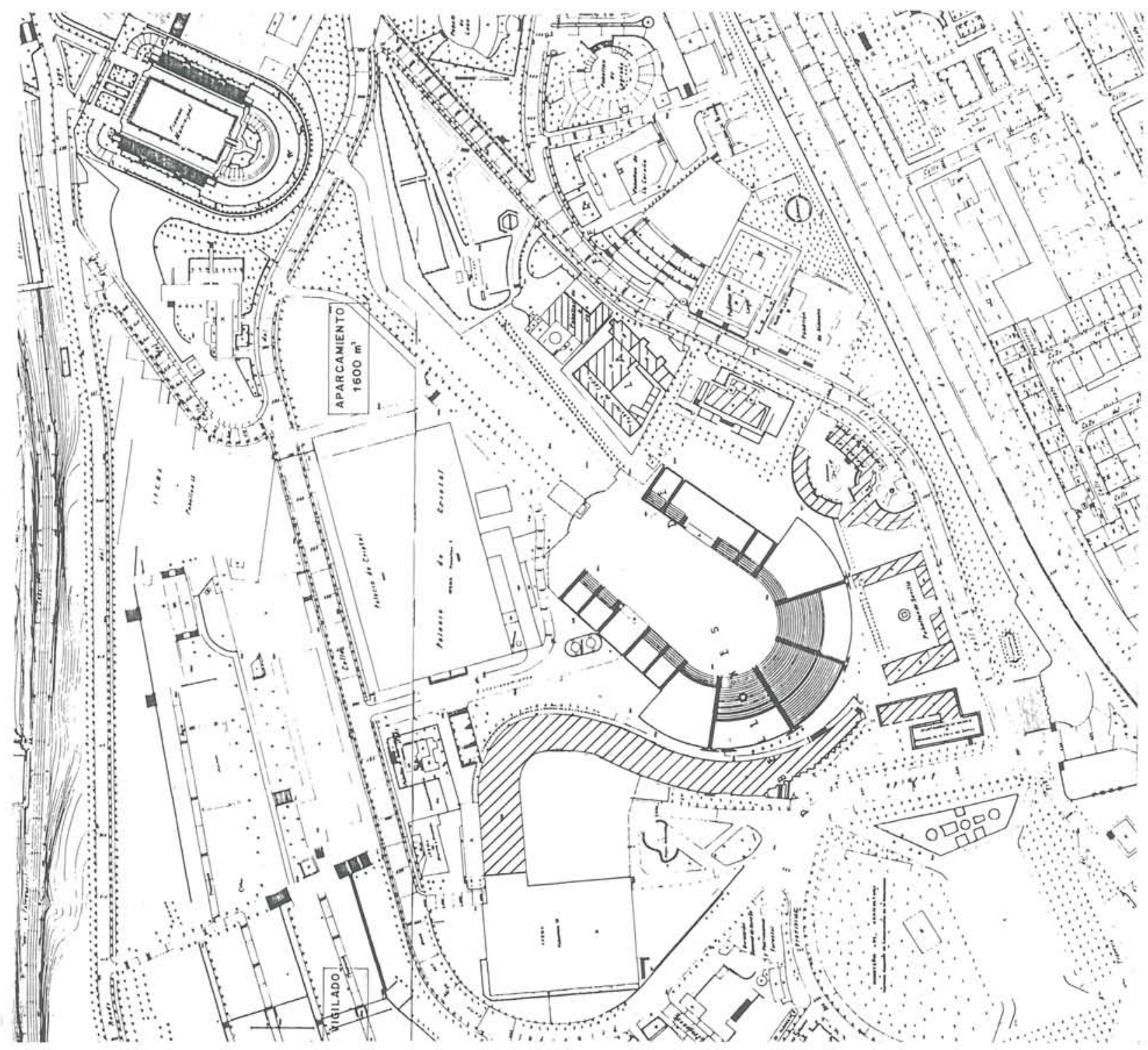

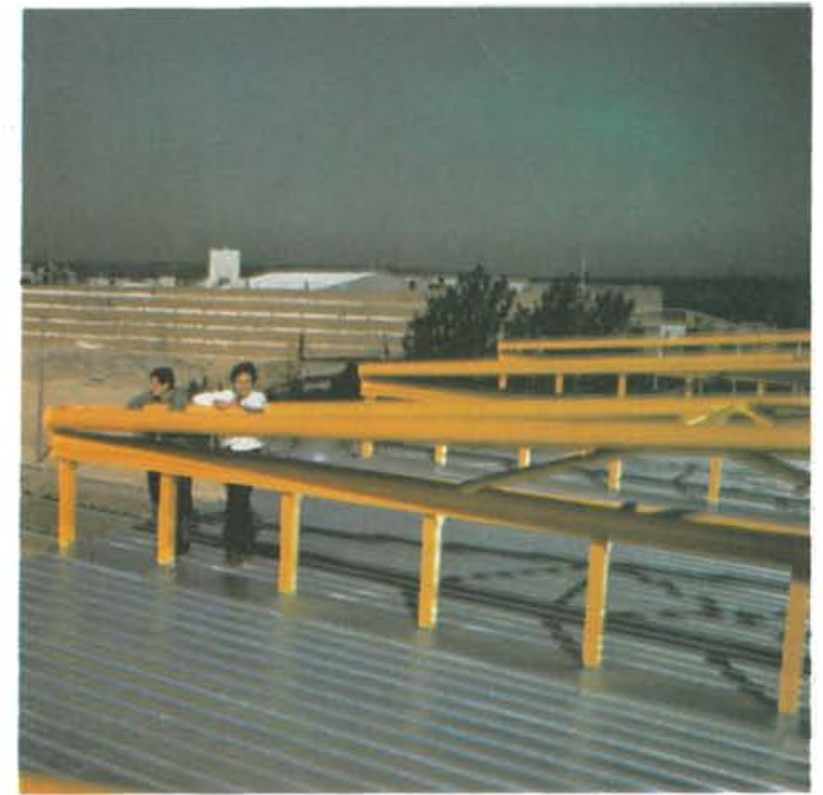

Aspecto de la cubierta colgada, montantes de cuelgue y vista del auditorio.
Entre las soluciones iniciales existia la del muro más o menos transparente concebido con una fábrica de ladrillo o de hormigón. La necesidad de colgar focos o equipos de sonido y la de disponer del escenario más amplio posible nos llevó al planteamiento de una viga de grandes dimensiones, que se constituia además como pasarela de servicios en los conciertos y peatonal en tiempo fuera de ellos, salvando la vaguada del antiguo auditorio.

Era necesario resolver al mismo tiempo los problemas de acceso y evacuación de público, los problemas de carga y descarga en el escenario y la protección de este último frente a la lluvia. Asi como pensar en la construcción de unos camerinos, zonas de control, almacenes, y edificio de autoridades, transmisiones, videos, etc. Lo que puede diferenciar un auditorio de este tipo, frente a otro al aire libre más convencional es sin duda la importancia que adquieren los me- 


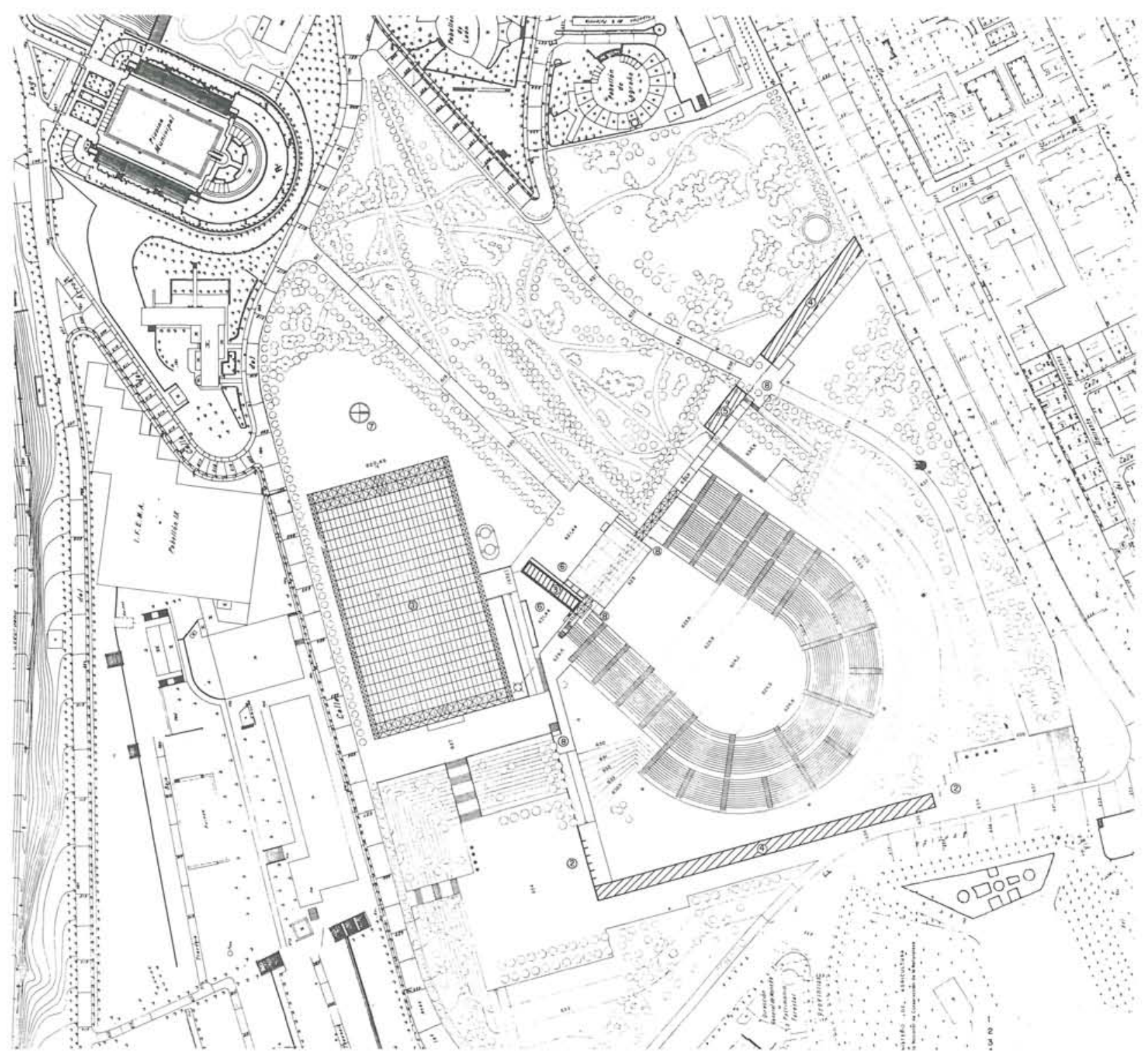

Situación y accesos (estado actual).

1. Palacio de Cristal. - 2. Accesos público. - 3. Edificio servicios artistas. - 4. Edificio servicios público. - 5. Bar-restaurante. -6. Aparcamiento. -7. Helipuerto. - 8. Evacuación.

dios técnicos y de difusión empleados, acordes con el seguimiento masivo de público antes mencionado.

En la actualidad sólo se encuentra construida una parte del programa, que atiende al escenario descrito, concebido como una plataforma de hormigón sobre la que se lanza la gran viga de instalaciones. Sobre la viga y la plataforma del escenario se ha construido una marquesina de acero y chapa galvanizada que cubre el conjunto. Las dimensiones fundamentales se hallan reflejadas en los planos. Basta decir aqui que la

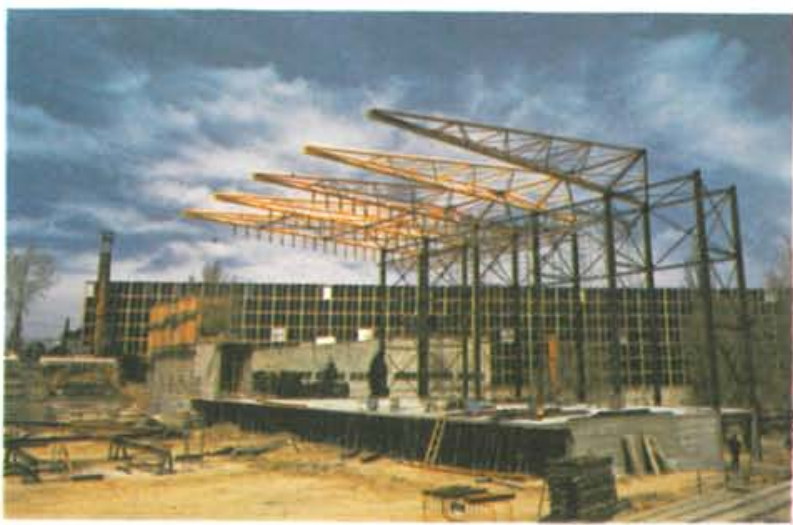

Ménsulas de la marquesina en fase de montaje. El pabellón de cristal, de F. Cabrero, al fondo. Forjado de escenario. 


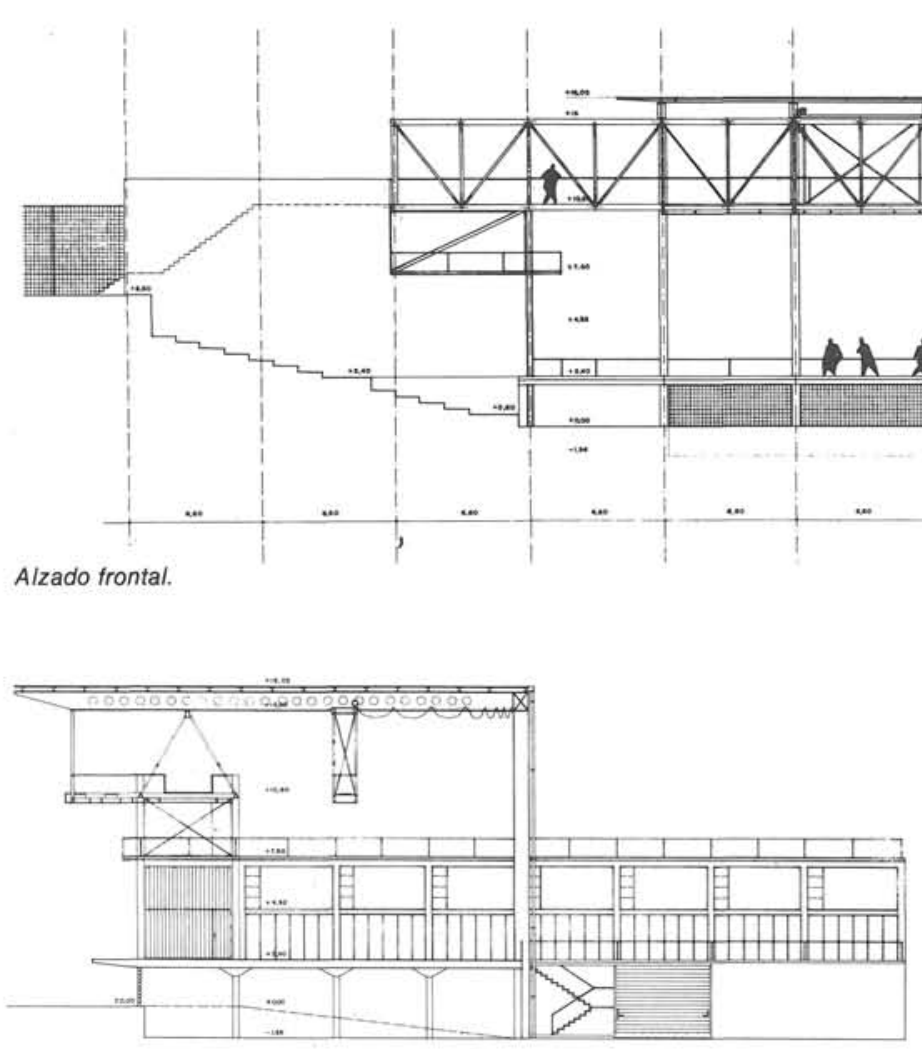

Sección transversal S-2.

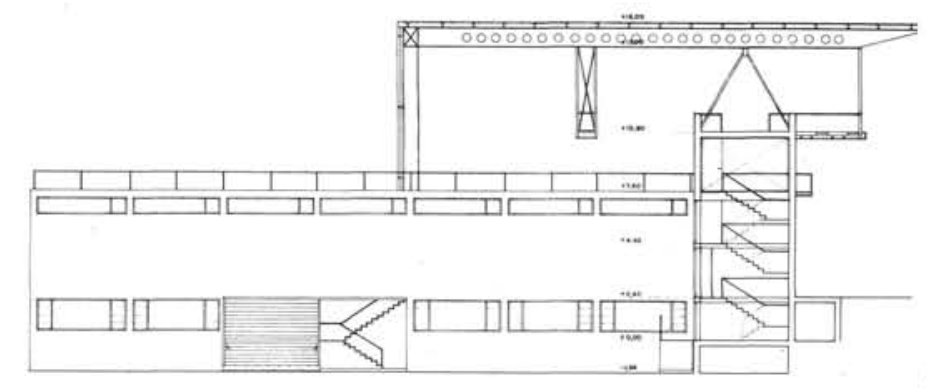

Sección transversal S-3. solución inicial

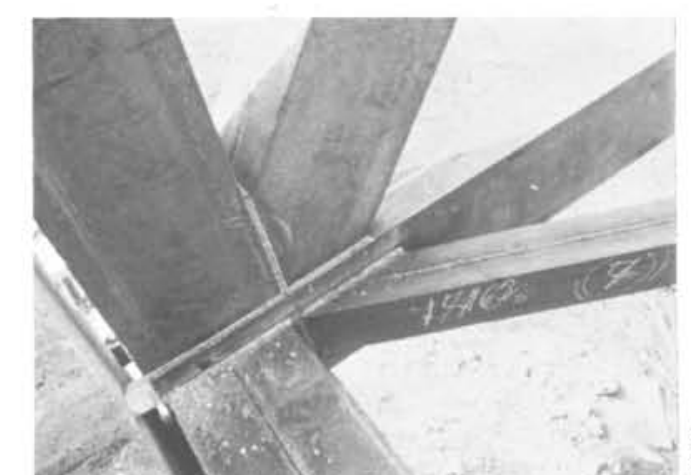

Pasarela. Nudo de apoyo sobre neopre-

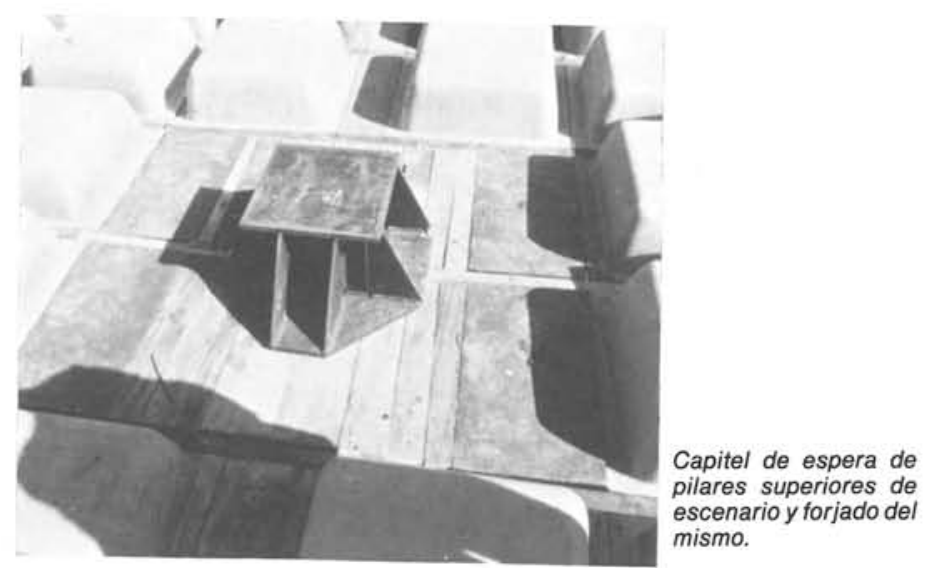




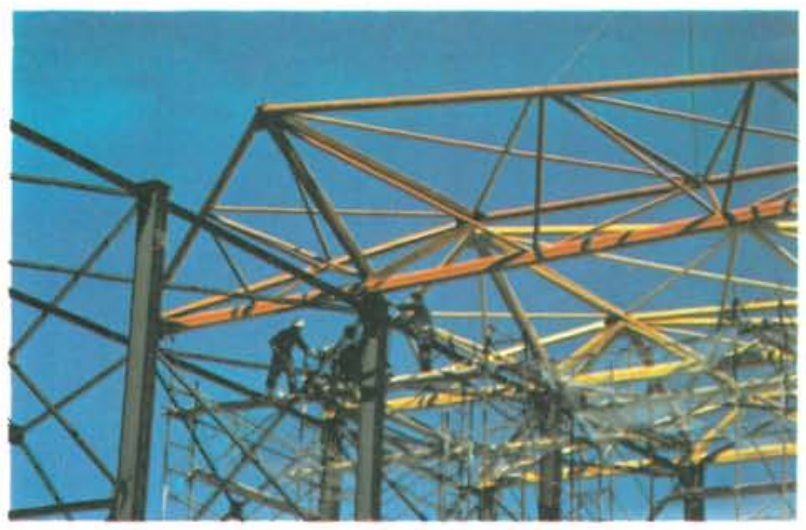

Fijación y pintura de las piezas de cubierta. Se aprecian las dos lineas de pilares. viga salva una luz de $79,2 \mathrm{~m}$, construida en acero con un diseño clásico de viga triangulada tipo Warren dimensionando las secciones de acuerdo con las solicitaciones previstas.

Los apoyos se han resuelto con piezas de neopreno de $210 \times 210 \mathrm{~mm}$, colocadas sobre los estribos de hormigón en los que se han situado los cuadros eléctricos, la cabina de control, y otras zonas auxiliares del escenario.

El conjunto es pues como un puente que pasa y atraviesa el espacio inferior de una gran marquesina. Un puente con los estribos habitables. Unos estribos de vidrio en donde se sitúa la cabina de control.

La construcción del conjunto se pudo realizar en un tiempo muy corto, apenas dos meses, gracias a una buena planificación de la obra, a la dedica-

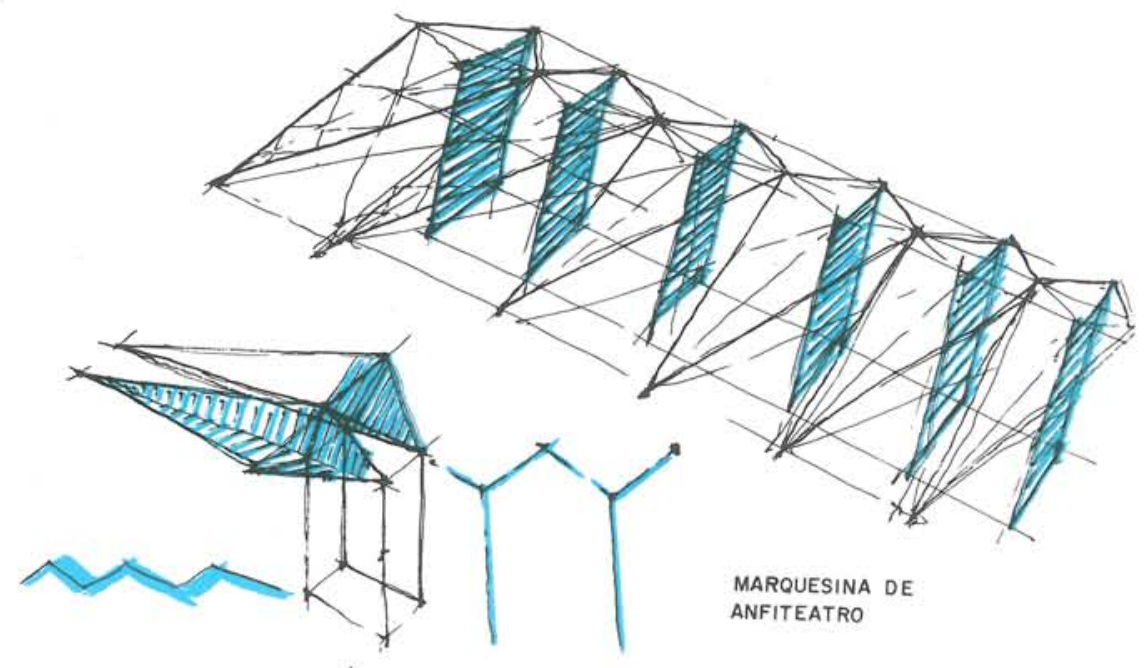

Esquema de funcionamiento estructural.

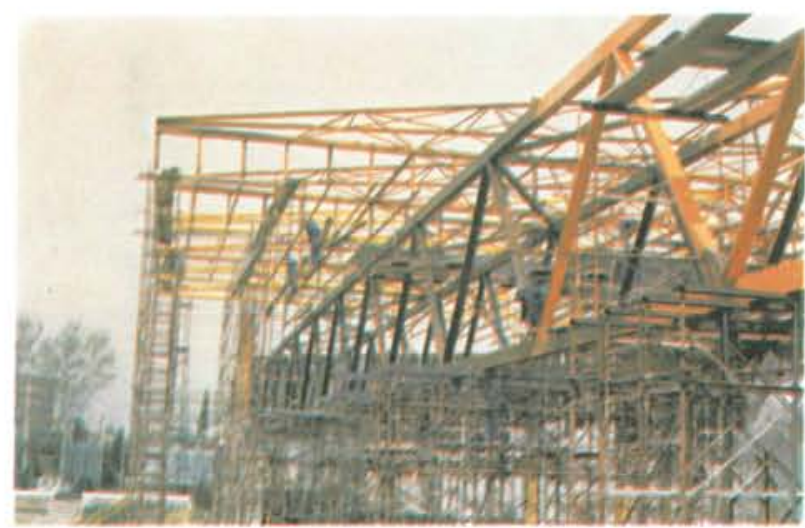

Fase general de montaje del conjunto. ción de la empresa constructora y a la caracteristica del proyecto que permitió realizar contemporáneamente la marquesina y la pasarela. Las piezas de la marquesina se construyeron en el suelo y se izaron posteriormente con grúa fijándose a los pilares en dos puntos con tornillo de alta resistencia. La concepción de la marquesina con una sección triangular en disminución permitia la posterior fijación de pequeños montantes desde los que era posible colgar la cubierta pensada como un conjünto de dos láminas de chapa galvanizada que contienen en su interior las correas necesarias y los elementos de arriostramiento.

La pasarela se transportó en cinco partes de distinta modulación, siendo la pieza mayor de tres módulos (cada módulo es de $6,60 \mathrm{~m}$ ), siendo el total de la pasarela de 12 módulos. El acero en 


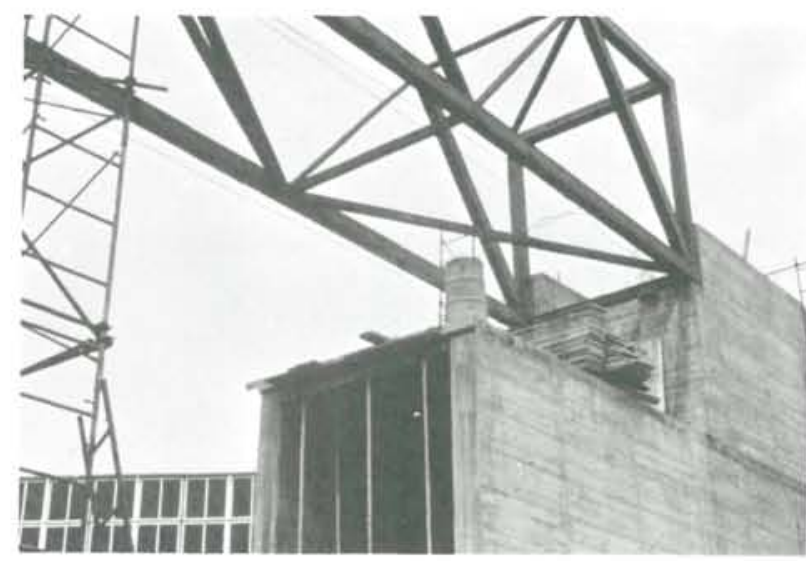

Montaje de pasarela sobre estribo izquierdo.

ambas piezas del tipo $\mathrm{A}-42 \mathrm{~b}$ fue soldado en todas las uniones menos en las descritas de la marquesina. Los problemas del plazo y las muy dificiles condiciones atmosféricas que presidieron la realización de la obra obligaron a reforzar posteriormente las uniones concebidas a tope en los puntos de unión de los cordones de la pasarela formados por dos $\mathrm{U}$ del 300 con dos chapas interiores de $250 \times 15$ a modo de collarin que hace de fondo de cordón.

La ejecución fue sin duda excelente a pesar de las difíciles condiciones de trabajo, no produciéndose ningún problema digno de mención. Todas las piezas, la pasarela y la marquesina fueron fabricadas con correcciones de tipo geométrico para evitar efectos ópticos desagradables. Asi la pasarela se montó con una contraflecha de $300 \mathrm{~mm}$, que se redujo en unos $10 \mathrm{~cm}$ al descimbrarla. La marquesina también tiene unas ligeras curvaturas de la lámina de chapa acortando los montantes de cuelgue en el centro de la misma unos diez cm.

Los planos que reproducimos corresponden a los primeros concebidos y los que reflejan la solución construida. Las diferencias entre unos y otros son notables; quizás la más importante sea la diversa sección de la pasarela al inicio triangular y posteriormente en forma de cajón para dar una mayor superficie de trabajo y para distribuir las compresiones en dos cordones.

Otra de las variaciones apreciables está en la concepción de la marquesina, en un principio apoyada sobre la pasarela y después en vuelo. Las razones son varias: una la necesidad de no hipotecar su construcción a la de la pasarela, otra el deseo de techar de un modo homogéneo el escenario con una lámina única. Esta opción nos llevó al diseño hoy construido con montantes de cuelgue de las láminas de cubierta y a la sección triangular de las piezas que permiten una muy cómoda rigidización entre si de todo el conjunto.

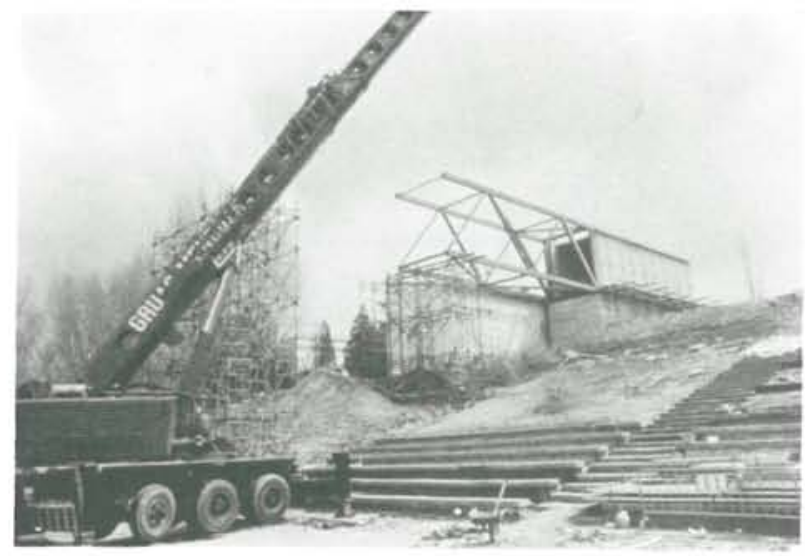

Estribo derecho. Fase de montaje del primer tramo de pasarela.

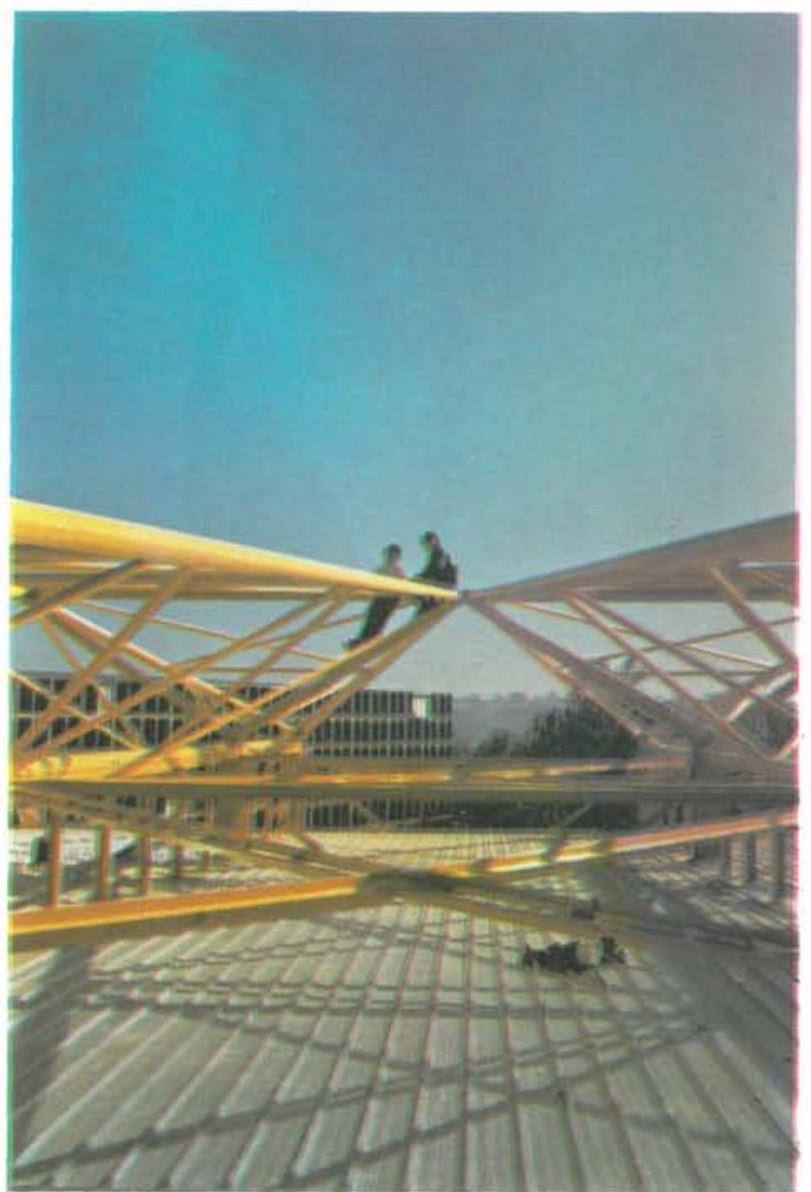

Ménsulas de cubierta terminadas. Se aprecia la cota con el pabellón de F. Cabrero al fondo y la lámina de cubierta colgada. 


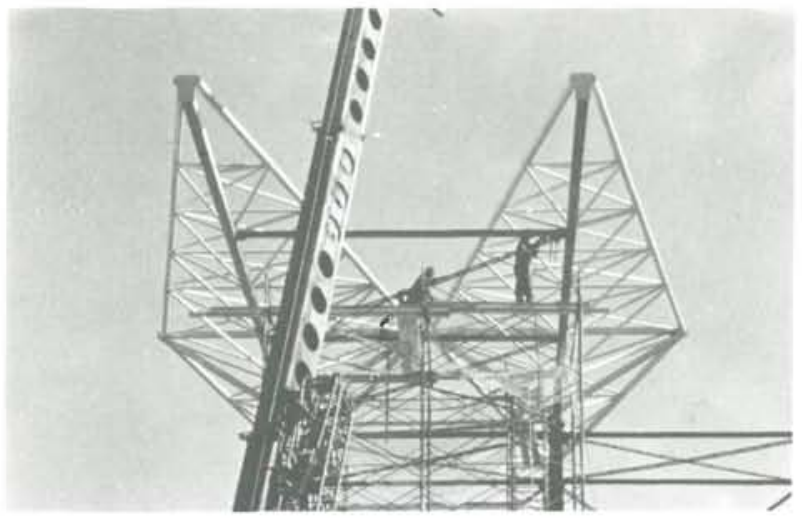

Montaje de las mensulas elevadas como piezas enteras despues de su fabricación en el auditorio.

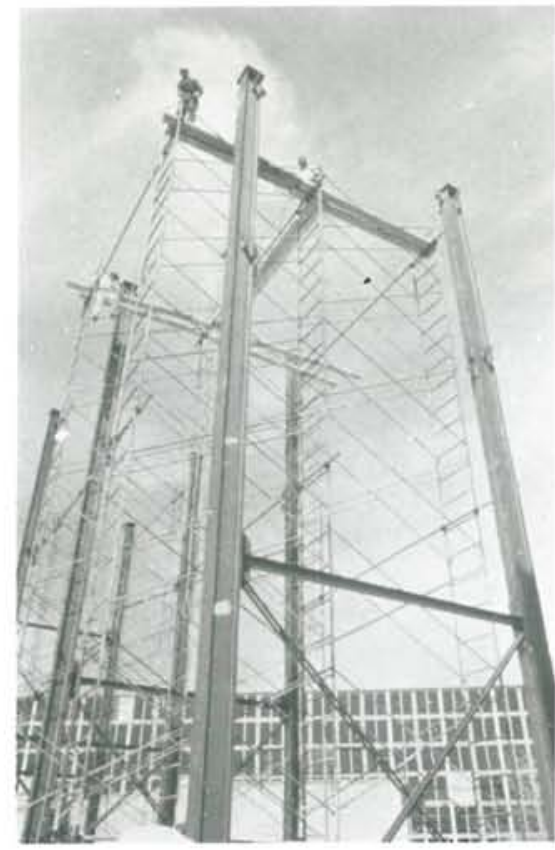

Montaje de las dos lineas de pilares de apoyo de cubrerta.

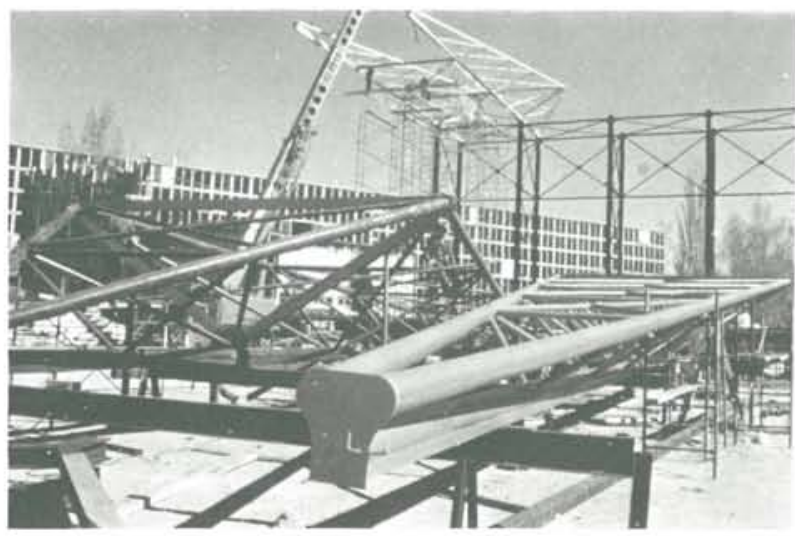

Fabricacion de las mensulas en el terreno; dos montadas al fondo.

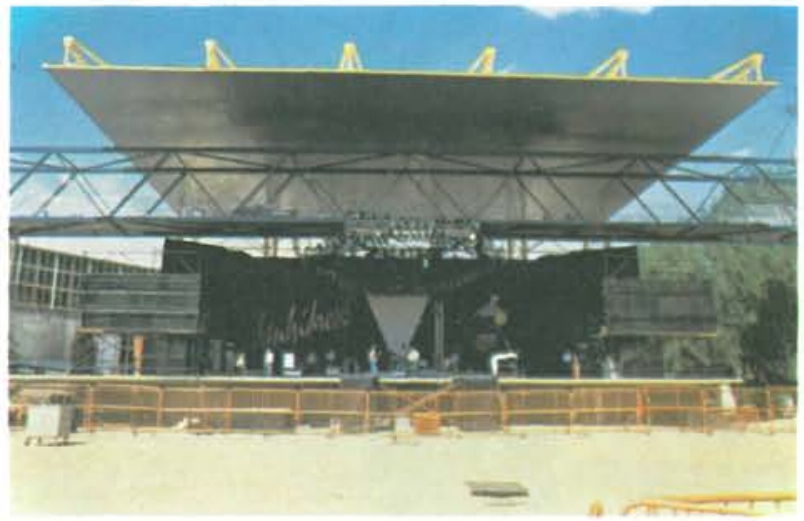

El resto de las soluciones apuntadas en los primeros dibujos se han respetado o serán construidas en breve. El éxito alcanzado en los primeros conciertos no puede ocultar que en la actualidad es preciso completar todos los elementos pensados, como son sin duda la construcción de los edificios auxiliares de camerinos, zonas de prensa y autoridades, una mejor regulación de accesos y servicios, que tambièn es preciso construir, sin olvidar la necesidad de completar las gradas tal y como está previsto en el proyecto.

Datos del proyecto:

$\begin{array}{ll}\text { Arquitectos } & \begin{array}{l}\text { Salvador Pérez Arroyo } \\ \text { Iñaki Abalos } \\ \text { Juan Herreros }\end{array} \\ \text { Estructura } & \begin{array}{l}\text { Enrique Bofill } \\ \text { Luis Agudo } \\ \text { J. Luis Garcia Rubio }\end{array}\end{array}$

Empresas Construcciones y Contratas Monoplex

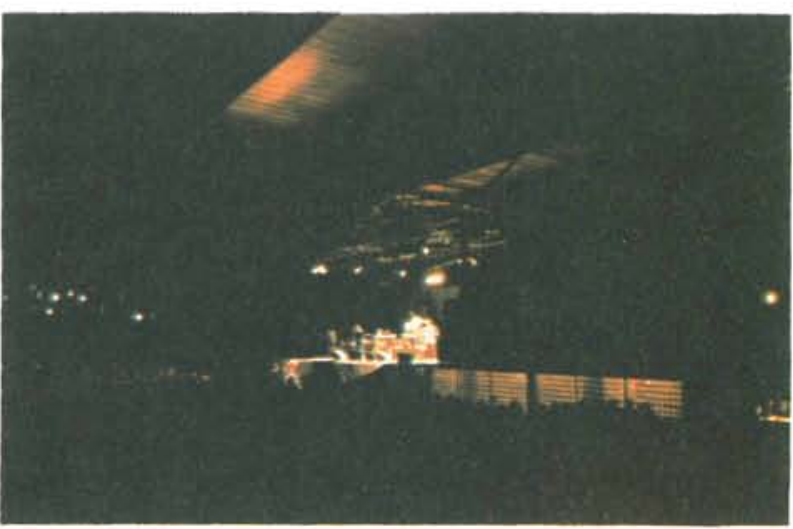

